\title{
Sensor defect detection in multisensor information fusion
}

\author{
Jan-Friedrich Ehlenbröker, Uwe Mönks, and Volker Lohweg \\ inIT - Institute Industrial IT, Langenbruch 6, 32657 Lemgo, Germany \\ Correspondence to: Jan-Friedrich Ehlenbröker (jan.ehlenbroeker@hs-owl.de)
}

Received: 15 October 2015 - Revised: 25 June - Accepted: 26 July 2016 - Published: 18 October 2016

\begin{abstract}
In industrial processes a vast variety of different sensors is increasingly used to measure and control processes, machines, and logistics. One way to handle the resulting large amount of data created by hundreds or even thousands of different sensors in an application is to employ information fusion systems. Information fusion systems, e.g. for condition monitoring, combine different sources of information, like sensors, to generate the state of a complex system. The result of such an information fusion process is regarded as a health indicator of a complex system. Therefore, information fusion approaches are applied to, e.g., automatically inform one about a reduction in production quality, or detect possibly dangerous situations. Considering the importance of sensors in the previously described information fusion systems and in industrial processes in general, a defective sensor has several negative consequences. It may lead to machine failure, e.g. when wear and tear of a machine is not detected sufficiently in advance. In this contribution we present a method to detect faulty sensors by computing the consistency between sensor values. The proposed sensor defect detection algorithm exemplarily utilises the structure of a multilayered group-based sensor fusion algorithm. Defect detection results of the proposed method for different test cases and the method's capability to detect a number of typical sensor defects are shown.
\end{abstract}

\section{Introduction}

A sensor, which is acquiring signals in an application, is generally assumed to be operating correctly. Sensors can nevertheless fail and do so during typical operation. Failure causes include improper handling, wear and tear, or random failure. The failure may on the one hand be a complete failure of the sensor, which is easily detectable, as the sensor stops delivering any data. On the other hand, partial defects are more difficult to detect: if a sensor continuously delivers values, it is neither directly detectable, nor decidable if the sensor measurements are valid or not. In case of partial defect, the sensor might produce values that deviate more from the true value than their given accuracy. This is problematic for condition monitoring purposes in manufacturing processes. Here, sensor defects lead to a decrease in product quality or a reduction in the produced quantity of a given product. Depending on the sensor's use case, a sensor defect has possibly even more severe consequences.
There are multiple possible ways to detect and handle sensor defects. An overview over the most important methods for detecting sensor faults is given in the following.

Simple approaches use rule-based threshold systems to detect sensor faults. For example in Sharma et al. (2010) the standard deviation of a sensor measurement within a window is used to detect sensor noise and the rate of change of a sensor measurement to detect short peak errors.

Apart from the aforementioned simple approaches, most algorithms are more complex and use statistical measures, machine learning methods or a combination of both.

Examples of statistical methods that are used for sensor fault detection are principal component analysis (PCA; Kerschen et al., 2005) and linear discriminant analysis (LDA; Helwig et al., 2015). In Kerschen et al. (2005) PCA is used to detect sensor faults differences between a reference measurement and live measurements for linear systems. The approach used in Helwig et al. (2015) utilises the data of multiple sensor fault states and a fault-free state to generate an LDA space, which is a space reduced in dimensional- 
ity that allows for a linear separation between the different fault states and the non-faulty state. More complex PCAbased methods use dynamic PCA-based approaches for sensor fault detection, as for example in Hu et al. (2012), where a self-adapting PCA-based method is used. For the detection of sensor defects in non-linear systems, PCA-based methods that use kernel functions are proposed (Choi et al., 2005).

Bayesian belief network (BBN) based methods are proposed in Mehranbod et al. (2003, 2005). They model every sensor as its own multiple-node BBN. Training data are used to generate state probabilities (example states: very negative, negative, zero, positive, very positive) for the nodes and when there are deviations from these trained state probabilities a sensor error is detected.

Artificial neural networks (ANNs) (cf. e.g. Mattern et al., 1998; Xu et al., 1999; Zhu et al., 2012; Helwig et al., 2015) and approaches based on clustering (cf. e.g. Bay and Schwabacher, 2003; Kusiak and Song, 2009) to detect sensor faults. While clustering-based algorithms detect sensor faults with the help of outlier detection, ANNs are used to generate a fault-free sensor output via sensor correlation (Mattern et al., 1998; Xu et al., 1999) or to detect if the sensor state is faulty or fault free (Zhu et al., 2012).

One approach is the application of sensors that execute self-tests to detect the sensor performance and sensor defects. Depending on the type of sensor and the intended use, this may be a valid way to handle sensor defects. Nevertheless, self-testing capabilities are often limited to simple function tests. Additionally, such sensors have higher acquisition costs compared to sensors without self-test abilities: considering standard off-the-shelf sensors, additional electronics must be integrated to each sensor to facilitate selftesting. This requires additional engineering, hardware, and production costs, which are added on top of the original sensor price. Moreover, sensors with self-test abilities are not available for every use case, especially when applications impose special requirements, e.g. explosion protection.

The approach followed in this contribution is the application of multiple sensors for monitoring one and the same object or property. In such a use case, standard sensors can be applied with no additional acquisition costs. Their signals are collected, aggregated, and processed in a multisensor information fusion process. The fusion system serves for supervision of the monitored system and also of the applied sensors. Here, additional one-time costs for the engineering and acquisition of the fusion system apply instead of increased costs for each sensor. In addition, retrofitting of existing applications is facilitated in this way. Then already applied sensors are utilised for fusion, which is enriched by defect detection. The fundamentals of such a multisensor information fusion approach are described in the following section.

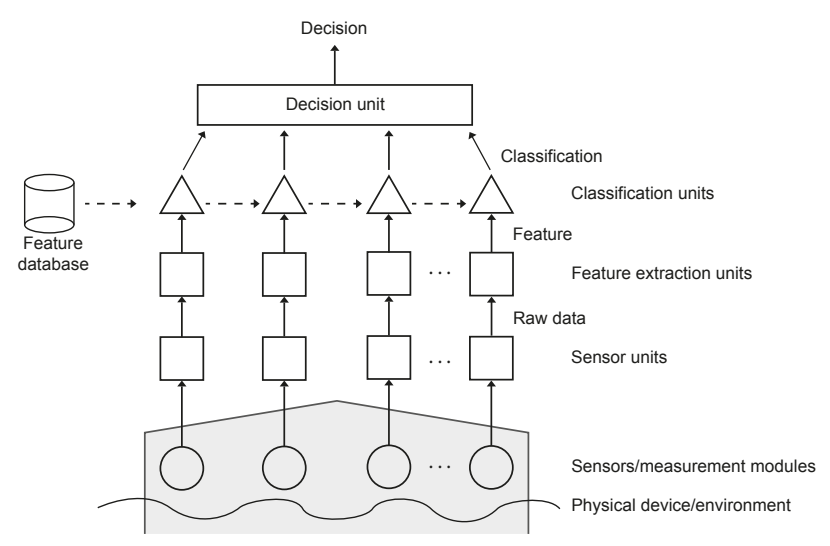

Figure 1. Multimodal system.

\subsection{Multisensor information fusion}

Many recent systems are based on one main sensory apparatus. They rely on the evidence of a single source of information (e.g. photodiode scanners in vending machines, greyscale cameras in inspection systems). These systems, called unimodal systems, have to contend with a variety of general difficulties. According to Ross and Jain (2005), these are raw data noise, intraclass variations, interclass similarities, and non-universality. Some of these mentioned limitations are overcome by the inclusion of multiple information sources. Such systems, known as multimodal systems, are expected to be more reliable due to the presence of multiple, partly signal-decorrelated, sensors. They address the problems of non-universality and, in combination with meaningful interconnection of signals (fusion), the problem of interclass similarities. At least, they can inform the user about problems with intraclass variations and noise. A generic multimodal system consists of four important units (cf. Fig. 1): (i) the sensor unit, which captures raw data from different measurement modules (i.e. sensors); (ii) the feature extraction unit, which extracts an appropriate feature set as a representation for the system, from which the raw data are captured; (iii) the classification unit, which compares the current features to their corresponding features stored in a database; (iv) the decision unit, which uses the classification results to determine whether the obtained results represent, e.g., a safe state of a hazardous material store (Lohweg and Mönks, 2010b).

The basic information fusion concept relies on the fact that the lack of information supplied by sensors is completed by the fusion process. It is assumed that, for example, two sensors $\left(S_{1}\right.$ and $\left.S_{2}\right)$ with different active physical principles (e.g. pressure and temperature) are connected in a certain way. With fusion of different sources the perceptual capacity and plausibility of a combined result should be increased. Furthermore, the resulting information should in some sense be better than in the case where the sources are used individually. The common resulting effect is the generation of infor- 
mation, which is more dense and of higher quality than every single data source (Luo and Kay, 1989) and thus a decrease of the result's inherent uncertainty.

A simple way to process multiple observations in an information fusion sense is a threshold system. Every sensor observation is classified individually, based on a threshold. These results are fed into a majority voting system to generate a global decision about a status (Alpaydin, 2010). This approach may decrease the impact of a sensor defect, as multiple non-defective sensors overrule one defective sensor. Nevertheless, such systems are too simple to model a complex application sufficiently. Hence, more adequate (but also more complex) multisensor information fusion algorithms are used for the processing of sensor observations and the robust determination of a system's state. These methods are known in the literature for many years (Khaleghi et al., 2011; Hall and Llinas, 2001a). This field has gained increasing attention starting in the 1970 s when new sensors, advanced processing techniques, and increasingly powerful processing hardware became available. Starting then, appropriate data processing models and fusion algorithms have been driven nearly exclusively by applications in the military defence sector. During the 1990s and early 2000s, those algorithms have been adopted by the civil sector for applications in industrial fault diagnosis and condition monitoring applications (Hall and Llinas, 2001a). A current fusion definition was introduced by Steinberg and Bowman (2001):

Definition 1. Information fusion (Steinberg and Bowman, 2001, p. 2-4): "[the] process of combining data or information to estimate or predict entity states."

Fusion is possible at three distinct levels (Hall and Llinas, 2001b). At signal level, sensor signals are combined. It is necessary that the signals are comparable in the sense of data amount, i.e. sampling rate (adaption), dimension, registration, and time synchronisation. If this constraint cannot be fulfilled, fusion on any of the following two levels is appropriate. At feature level, signal descriptors (features) are combined. Human cognitive functions rely on this association principle for recognition tasks. At symbol level, classification results are combined. This happens either after obtaining all individual decisions per sensor, or on top of a number of features or signal level fusion steps. The degree of abstraction increases from signal level to symbol level, whereas the fusion itself is more efficient with increasing abstraction. Nevertheless, additional processing steps in advance to fusion might increase the overall complexity.

Besides, Ross and Jain (2005) state that fusion at an early processing stage is usually more effective than at a later stage, since input signals or features contain more information about the physical data than score value outputs of classifiers. High abstraction level fusion is less effective also due to the fact that data reduction methods are applied in the intermediate steps resulting in information loss (cf. Hall and Llinas, 2001b).

\subsection{Related work}

New concepts of distributed intelligent sensors have recently been introduced, in which an intelligent sensor is defined to be a system equipped with communication and processing capabilities, and acquires data from several elementary sensors attached to it (Duquet, 2015). Such concepts and architectures pose challenges to the design and operation of distributed monitoring systems (Mönks et al., 2015), among which is the handling of conflicts between sensor observations during operation: conflict occurs whenever information bear evidence for not only one opinion/proposition, but also for another. This might either be due to actual failure in the observed process or system, or caused by one or more defective sensors. The latter case is the most severe one, since wrong decisions might be derived if sensors were considered reliable, although they are not.

Conflict handling is to a certain extent independent from the model applied to represent the information: while probability theory (Jaynes, 2003; Bishop, 2009) and possibility theory (Zadeh, 1978; Dubois and Prade, 1993) need to incorporate further processing steps for conflict handling, the Dempster-Shafer theory of evidence (DST) (Dempster, 1967; Shafer, 1976) is inherently designed to handle conflicts. Nevertheless, the DST has shown to bear defects with respect to high-conflicting situations (cf. e.g. Zadeh, 1986; Yager, 1987).

A conflict-handling data fusion algorithm, based on the DST and improving its deficiencies, is the multilayer attribute-based conflict-reducing observation (MACRO) system (Mönks and Lohweg, 2013, 2014). Its fusion algorithm has shown good performance, especially in situations, where the input data are conflicting (Mönks et al., 2012). It nevertheless offers no direct way for the fusion algorithm to detect defective sensors. The situation is similar for other sensor fusion approaches like Bayes' theorem in the scope of probability theory (Bishop, 2009), Dempster's rule of combination in the scope of DST (Shafer, 1976), or ordered weighted averaging (OWA) aggregation (Yager, 1988) in the scope of fuzzy set theory (Zadeh, 1965). They are all standard and widely applied fusion approaches, which do not offer inherent defect detection methods.

There are approaches in the literature for the detection of process anomalies by using sensor fusion methods, including methods that handle or reduce conflicts between sensor observations (Khaleghi et al., 2011). Almost no sensor fusion algorithm exploits the conflict between sensor measurements or consistency measures as a method to predict or detect defective sensors. One detection method for defective sensors based on conflict is proposed in Ricquebourg et al. (2008). Background of this method is the computation of a conflict factor between pairs of sensors. The conflicts are analysed and classified concerning the duration and intensity of a conflict so that in the end every sensor has a dedicated state (fail- 
ure, no failure). During sensor fusion, the state of a sensor is used to exclude sensors that have a failure state.

Another exception is Krüger (2015), where conflicts in Bayesian networks are used to detect sensor failures. In this work four approaches for sensor failure detection are described. Two of the described approaches are based on binary conflicts (conflict present or no conflict present), while the other two approaches utilise a gradual conflict measure that shows the actual level of conflict. For the approaches with binary conflicts the frequency of conflicts is used as a measure for defect detection. The algorithms based on gradual conflicts use the mean gradual conflict value for defect detection. The final defect detection is carried out with the help of detection thresholds. Furthermore, all approaches described in Krüger (2015) use an adjustable sliding test-window, which incorporates multiple classification cases for defect detection.

Other sensor fusion approaches incorporate sensor reliability or similar values into the fusion process. In Elouedi et al. (2004) a DST-based sensor fusion approach that incorporates a discounting factor for sensors is proposed. This discounting factor is computed based on the existing knowledge compared to the measurement of a sensor. The difference between the known class of the objects in a training data set and the assessment of a sensor is used to determine the discounting factor, with higher differences resulting in a higher discounting factor. The smaller the discounting factor, the more reliable the sensor considered.

The reliability computation method proposed by Martin et al. (2008) is also based on DST. It utilises the distances between a sensor's measurement and the combination of all other sensors' measurements to compute a conflict measure. The reliability value of a sensor is then in turn computed based on a decreasing function, which utilises the conflict measure. This results in a lower reliability value for sensors with higher conflict, which is used as a discounting factor during the sensor fusion process.

The approach presented in this article is partly based on Glock et al. (2011), which introduces a method to determine the reliability of sensors. This is in turn used to weight the sensors during a sensor fusion process.

In summary, sensor fusion approaches are available, which incorporate a form of reliability computation for sensors. Their outcome is applied to weight sensors during the sensor fusion process (Elouedi et al., 2004; Martin et al., 2008; Glock et al., 2011). Only a few methods use sensor fusion approaches to actually detect sensor defects (Ricquebourg et al., 2008; Krüger, 2015).

This article proposes a method that uses group-based structures where the sensor defects are computed based on groups of sensors instead combining all sensors at once, as other approaches do.

\subsection{Structure}

In this paper, a method is proposed that utilises the inherent multilayer group-based structure of MACRO and detects sensor defects with the help of sensor consistency computations. While the structure of MACRO is utilised in the presented approach, it is also applicable in other groupbased fusion approaches. Its effectiveness is demonstrated in the scope of the research project "itsowl-IGel" (itsowl-IGel, 2015). The main goal of itsowl-IGel is the development of a condition monitoring and early warning system for hazardous material stores, which safely contain materials like dangerous chemicals. No automatic monitoring mechanisms are legally demanded; hence, itsowl-IGel represents pioneering work in this area.

This paper is separated into the following sections: Sect. 2 presents a brief overview about the information fusion system MACRO, followed by a more detailed view into the method for sensor defect detection. The experiments and results are given in Sect. 3. The paper concludes with Sect. 4 by giving a discussion of the results and delivering an outlook on future work.

\section{Approach}

The approach section is divided into multiple parts: first a description of the applied information fusion system MACRO is presented. This subsection is followed by preliminary information for the following parts and subsections on sensor reliability and consistency computation. The contributed sensor defect detection approach, which combines a consistency-based reliability computation with the sensor fusion system, concludes the approach.

\subsection{Multilayer attribute-based conflict-reducing observation (MACRO)}

The MACRO approach is applied for the fusion of several sensor signal inputs. MACRO's structure is depicted in Fig. 2.

The basic multilayer structure of this approach is inspired by the decision-making process of groups of humans: individual humans (sensors) discuss their opinions (measurements) in groups (attribute layer). This group decisionmaking process includes conflicts. The information generated in the various groups is then combined on an organisational level (system layer) to make a global decision. For more information on the human group decision-making background of MACRO, the reader is referred to Mönks and Lohweg (2013). It was shown that the application of this approach for hazardous material store monitoring is beneficial compared to state-of-the-art installations (Ehlenbröker et al., 2014).

The MACRO fusion approach for the determination of a system's global state is carried out as follows: signals from 


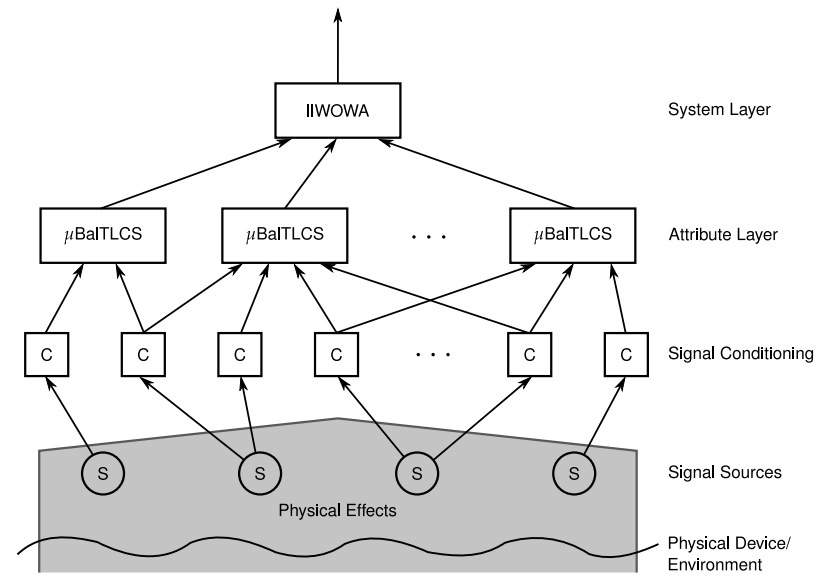

Figure 2. Multilayer attribute-based conflict-reducing observation system MACRO (Mönks and Lohweg, 2014).

the system as well as from its environment (like temperature) are acquired by sensors (signal sources). Features are extracted from the signals in the following signal conditioning step, which may also include signal preprocessing procedures. Multiple features may be extracted from one signal, as shown in Fig. 2, e.g. to determine the mean and variance from one signal. Without loss of generality, the following assumes one feature per signal.

In the signal conditioning step, the sensor measurements, which may include all sorts of (physically) different types of measurements (e.g. temperature, air pressure), are transformed into a unitless space. A fuzzy set theory (Zadeh, 1965) approach has been chosen for modelling the acquired data in a common unitless space between 0 and 1 . It is capable of model uncertainty in the data, which is coming from, e.g., sensor noise, and allow variations in the system's behaviour due to environmental changes (e.g. in temperature, humidity), which do not affect the fulfilment of the system's task. The Modified-Fuzzy-Pattern-Classifier (MFPC) (Lohweg et al., 2004) models the information by a unimodal potential function applied as fuzzy membership function $\mu: \mathbb{R} \rightarrow[0,1]$. This information model has proven its performance scientifically as well as in real-world applications (e.g. Lohweg et al., 2004; Niederhöfer and Lohweg, 2008; Mönks et al., 2010).

It employs an automatic learning procedure to determine the membership function based on measurement data:

Definition 2. Modified-Fuzzy-Pattern-Classifier learning (Lohweg et al., 2004; Mönks et al., 2010): the measurements of sensor $S_{i}$ at discrete time instance $k \in \mathbb{N}$ are denoted as $x_{i}[k]$. The vector $\boldsymbol{x}_{i}=\left(x_{i}[k]\right), 1 \leq k \leq N$ consists of the $N$ individual measurements. Then the measurements are represented by the Modified-Fuzzy-
Pattern-Classifier membership function as

$\mu\left(x, \boldsymbol{p}_{i}\right)=2^{-d\left(x, \boldsymbol{p}_{i}\right)}$

with $d\left(x, \boldsymbol{p}_{i}\right)=\left(\frac{\left|x-\bar{x}_{i}\right|}{C_{i}}\right)^{D_{i}}$.

The parameters $\boldsymbol{p}_{i}=\left(\bar{x}_{i}, C_{i}, D_{i}\right)$ are determined based on the measurement data $\boldsymbol{x}_{i}$ by

$\bar{x}_{i}=\frac{1}{N} \sum_{k=1}^{N} x_{i}[k], C_{i}=\frac{1}{2} \cdot\left(\max \left(\boldsymbol{x}_{i}\right)-\min \left(\boldsymbol{x}_{i}\right)\right)$.

The integer-valued parameter $D_{i}$ is chosen empirically, typically as a power of 2 to keep computation of Eq. (1) hardware efficient.

Hence, the parameter vector $\boldsymbol{p}$ defines the membership function's properties: $\bar{x}$ is its mean value, $C$ denotes the width, and $D$ determines the steepness of the membership function. Since $\boldsymbol{p}$ is automatically determined on the basis of measurement data, the current condition is encoded in the parameters. In order to actually represent the normal condition of the monitored system by $\boldsymbol{p}$, the measurement data must be acquired within a period of time, in which the system is operated in normal condition, which must be verified manually by a human expert, e.g. an experienced machine operator. Then the membership function is denoted as ${ }^{\mathrm{N}} \mu$. Please note: the fusion approach poses one demand on the applied sensors: the signals should be compatible to those during normal condition if the system does not change its behaviour, whereas they need to change if the system changes its behaviour. Whether the sensors output signals represent the ground truth is irrelevant in this scope; hence, calibration is not necessary. Instead, the reaction on changes is important.

The fuzzy membership function is applied to compute the grade of membership ${ }^{\mathrm{N}} \mu_{i}(x)={ }^{\mathrm{N}} \mu_{i}\left(x, \boldsymbol{p}_{i}\right)$, to which a sensor's measurement $x$ represents the normal condition. Note that one membership function is utilised per sensor $S_{i}$. An exemplary membership function for a temperature sensor is shown in Fig. 3.

MACRO then combines ensembles of conditioned signals in groups denoted as attributes. They represent certain properties or physical parts of the observed system, such as air quality, ventilation, or air conditioning. Hence, each attribute has a semantic meaning, which relates it to the physical system. The attributes are application dependent and manually defined during the fusion system design process. Redundancies, which occur by combining at least two information sources to one attribute, are exploited for both (i) detecting sensor faults and (ii) cross-checking the consistency of sensor values. The latter is carried out implicitly by the psychologically inspired fuzzified balanced two-layer conflict 


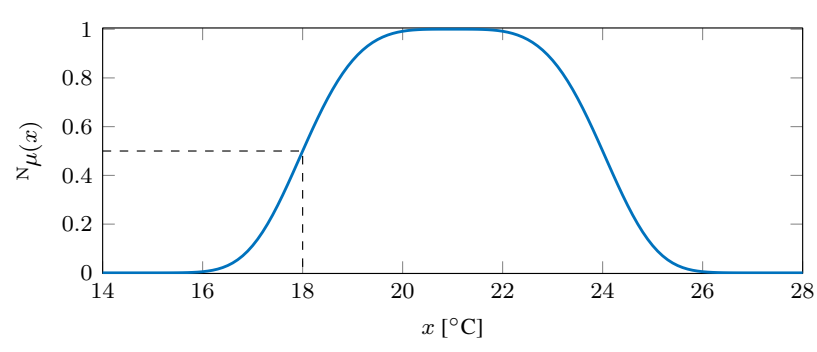

Figure 3. Exemplary membership function for the normal condition of a temperature sensor with $\bar{x}=21^{\circ} \mathrm{C}, C=3{ }^{\circ} \mathrm{C}$, and $D=4$. A measurement of $x=18^{\circ} \mathrm{C}$ results in a membership of $\mu(x)=$ 0.5 , as marked by the dashed lines.

solving ( $\mu$ BalTLCS) fusion approach (Lohweg and Mönks, 2010a; Mönks et al., 2012; Mönks and Lohweg, 2013). It creates one output ${ }^{\mathrm{N}} \mu_{a}:[0,1]^{n} \rightarrow[0,1]$ per attribute $a$ from its inputs. It additionally assigns each attribute an importance measure $I_{a} \in[0,1]$. The importance measure is based on the conflict between the sensors' individual opinions ${ }^{\mathrm{N}} \mu_{i}$. Hence, the higher the conflict between an attribute's inputs, the lower this attribute's importance. The importance are employed in the subsequent fusion of the attributes' opinions ${ }^{\mathrm{N}} \mu_{a}$, which are aggregated on a system level using the implicative importance-weighted ordered weighted averaging (IIWOWA) operator (Larsen, 1999) to reason about the entire system under supervision.

This operator is based on the importance-weighted ordered weighted averaging (IWOWA):

Definition 3. Importance-weighted ordered weighted averaging (Larsen, 1999): let $\boldsymbol{\mu}=\left(\mu_{1}, \mu_{2}, \ldots, \mu_{n}\right)$ be a vector of fuzzy memberships, and $\boldsymbol{I}=\left(I_{1}, I_{2}, \ldots, I_{n}\right)$ a vector of corresponding importance weights. The vector of weights $\boldsymbol{w}=\left(w_{1}, w_{2}, \ldots, w_{n}\right)$ determines whether the operator behaves more like the maximum or more like the minimum aggregation (more optimistic or more pessimistic), with

$\sum_{j=1}^{n} w_{j}=1$ and $\rho(\boldsymbol{w})=1-\frac{1}{n-1} \sum_{j=1}^{n}(n-j) \cdot w_{j}$

where $\rho(\boldsymbol{w}) \in[0,1]$ determines the aggregation's andness degree. This is a measure indicating to which degree the operator behaves like the minimum operation. An andness of $\rho(\boldsymbol{w})=0$ represents a pure maximum, $\rho(\boldsymbol{w})=1$ a pure minimum operation. The operator is able to model any degree of andness between $\rho=1$ and $\rho=0$ (Yager, 1988). Then the class of IWOWA operators is defined as

$h_{\mathrm{IWOWA}}(\boldsymbol{I}, \boldsymbol{w}, \boldsymbol{\mu})=\sum_{j=1}^{n} w_{j} \cdot b_{(j)}$, with $j \in \mathbb{N}_{n}=\{1,2, \ldots, n\}, \quad$ and $\quad b_{j}=\rho(\boldsymbol{w})+I_{j}$. $\left(\mu_{j}-\rho(\boldsymbol{w})\right)$, where $(\cdot)$ denotes a permutation on $\boldsymbol{b}$ with $b_{(1)} \geq b_{(2)} \geq \ldots \geq b_{(n)}$, i.e. the importance-weighted memberships sorted in decreasing order.

Larsen (1999) showed in Larsen (1999) that the class of IWOWA operators is order equivalent to the weighted arithmetic mean (WAM) operator. Order equivalence is sufficient when the operator is applied to provide preference ordering (Larsen, 2002). However, in situations where the aggregated value is used for other purposes, such as information fusion, full value equivalence to WAM is necessary. This property is obtained by normalising Eq. (3) in the interval of $h_{\text {IWOWA }}(\boldsymbol{I}, \boldsymbol{w}, \mathbf{0})$ and $h_{\text {IWOWA }}(\boldsymbol{I}, \boldsymbol{w}, \mathbf{1})$. This leads to the following class of operators:

Definition 4. Implicative importance-weighted ordered weighted averaging (Larsen, 2002): let $\mathbf{0}=(0, \ldots, 0)$ be a vector of zeros and $\mathbf{1}=(1, \ldots, 1)$ a vector of ones, each of length $n$. Then the class of IIWOWA operators is defined with Eq. (3) as

$$
\begin{aligned}
& h_{\text {IIWOWA }}(\boldsymbol{I}, \boldsymbol{w}, \boldsymbol{\mu}) \\
= & \frac{h_{\text {IWOWA }}(\boldsymbol{I}, \boldsymbol{w}, \boldsymbol{\mu})-h_{\mathrm{IWOWA}}(\boldsymbol{I}, \boldsymbol{w}, \mathbf{0})}{h_{\mathrm{IWOWA}}(\boldsymbol{I}, \boldsymbol{w}, \mathbf{1})-h_{\mathrm{IWOWA}}(\boldsymbol{I}, \boldsymbol{w}, \mathbf{0})} .
\end{aligned}
$$

In the scope of MACRO, the result of $h_{\text {IIWOWA }}\left(\boldsymbol{I}, \boldsymbol{w},{ }^{\mathrm{N}} \boldsymbol{\mu}\right)$ is denoted system health ${ }^{\mathrm{N}} h$, with ${ }^{\mathrm{N}} \boldsymbol{\mu}=\left({ }^{\mathrm{N}} \mu_{1},{ }^{\mathrm{N}} \mu_{2}, \ldots,{ }^{\mathrm{N}} \mu_{A}\right)$ being the attribute's memberships obtained by $\mu$ BalTLCS fusion, and $\boldsymbol{I}=\left(I_{1}, I_{2}, \ldots, I_{A}\right)$ the corresponding importances.

Detailed information regarding MACRO and $\mu_{\text {BalTLCS }}$ is found in Mönks et al. (2012); Mönks and Lohweg (2013), while optimisations concerning an efficient implementation are found in Mönks and Lohweg (2014). This contribution concentrates on MACRO's fusion on the attribute layer. Here, the sensor signals are fused initially and checked for consistency.

\subsection{Monitoring of sensor reliability}

Sensors are utilised in real-world applications to acquire signals, which represent the current situation in the application. The IEEE standard 610-1990 defines "reliability" as follows:

Definition 5. Reliability (IEEE Computer Society, 1990, p. 170): "[t]he ability of a system or component to perform its required functions under stated conditions for a specified period of time."

A sensor's reliability determines the quality of such a mapping from the physical situation to the sensor's output signal.

Glock et al. (2011) proposed a method to monitor sensor reliability, which is followed in this contribution. It is defined by 
Definition 6. Sensor reliability measure (Glock et al., 2011): the sensor reliability measure of sensor $S_{i} \in \mathcal{S}, i \in \mathbb{N}_{n}$ is defined as

$$
r_{i}=\min \left(r_{i}^{\mathrm{s}}, r_{i}^{\mathrm{d}}\right)
$$

where $r_{i}^{\mathrm{s}}$ denotes a sensor's static and $r_{i}^{\mathrm{d}}$ its dynamic reliability.

Reliability is split in a static and a dynamic part. Static reliability $r_{i}^{\mathrm{s}}$ expresses the probability that the sensor operates correctly in general. Each sensor in a real-world application is exposed to external, inevitable effects like ageing, which affects its output signal such that it deviates from the actual situation of the application. In consequence, the sensor's reliability is affected over time. This is represented by the dynamic part $r_{i}^{\mathrm{d}}$.

In order to compute the dynamic reliability, Glock et al. (2011) make use of the concepts of majority observation and consistency. In Glock et al. (2011), information fusion for machine condition monitoring is considered. It employs multiple sensors acquiring their signals from the same application. The sensor outputs are approximations of the true value and hence prone to uncertainty, which is determined by each sensor's characteristics. Therefore, each sensor's measurement is considered by

Definition 7. Sensor observation (Glock et al., 2011): let $x_{i}$ be the output of sensor $S_{i}$. Then this measurement is represented by the possibility distribution $\pi_{i}: \mathbb{R} \rightarrow$ $[0,1]$, which is denoted as sensor observation and models the sensor output's characteristics for the given measurement $x_{i}$.

Based on this, the degree of consistency between individual observations is determined by

Definition 8. Consistency index (Glock et al., 2011): let $T \subseteq \mathcal{S}$ be a subset of sensors $S_{i} \in \mathcal{S}$ with their respective observation $\pi_{i}$. Then the consistency index of their observations is determined as

$$
h(T)=\sup _{x \in \mathbb{R}}\left(\min _{i \mid S_{i} \in T}\left(\pi_{i}(x)\right)\right),
$$

with $h(T) \in[0,1]$ for all $T$.

A geometric interpretation of the consistency index is the height of the overlapping parts of all considered possibility distribution functions, i.e. observations. In case the observations of the employed sensors $S_{i} \in T$ are on different measurement scales, these need to be transformed to a common scale by fuzzification, hence a mapping $\mu_{i}: x_{i} \rightarrow$ $[0,1]$. Such situations occur due to unequal dimensions (twodimensional image vs. one-dimensional force) or physical units (colour temperature in $\mathrm{K}$ vs. force in N). Thus, without fuzzification, the consistency index is not computable, whereas this measure is necessary to determine the majority observation. It is defined as

Definition 9. Majority observation (Glock et al., 2011): let $2^{\mathcal{S}}$ be the set of all subsets of $\mathcal{S}$. Then the set of sensors $\mathcal{S}_{\mathrm{m}}$, determined by

$$
\mathcal{S}_{\mathrm{m}}=\left\{T \mid \sup _{T \in \mathcal{L}^{\mathcal{S}}}(h(T)>0)\right\},
$$

forms the majority observation, if and only if $\left|\mathcal{S}_{\mathrm{m}}\right|>1$.

Considered geometrically, the observations of each member of $\mathcal{S}_{\mathrm{m}}$ overlaps with at least one other member of $\mathcal{S}_{\mathrm{m}}$. All of their observations are considered fully consistent and span the range of the majority observation.

Although the remaining sensors $\left\{\mathcal{S} \backslash \mathcal{S}_{\mathrm{m}}\right\}$ do not contribute to the majority observation, their observations are considered consistent to a certain degree. In order to quantify the consistency, Glock et al. (2011) proposed to relate the centres of gravity of each observation $\pi_{i}$ to the range of the majority observation:

Definition 10. Majority consistency measure (Glock et al., 2011): let $\pi_{i}$ be the observation of sensor $S_{i} \in \mathcal{S}$. It is defuzzified by the centre of gravity method (Klir and Yuan, 1995, p. 336):

$$
c\left(\pi_{i}\right)=\frac{\int_{-\infty}^{\infty} \pi_{i}(x) \cdot x \mathrm{~d} x}{\int_{-\infty}^{\infty} \pi_{i}(x) \mathrm{d} x} .
$$

The range of the majority observation $\left[c_{\mathrm{m}}^{\min }, c_{\mathrm{m}}^{\max }\right]$ is determined over the respective observations' centres of gravity by

$$
c_{\mathrm{m}}^{\min }=\min _{i \mid S_{i} \in \mathcal{S}_{\mathrm{m}}}\left(c\left(\pi_{i}\right)\right), c_{\mathrm{m}}^{\max }=\max _{i \mid S_{i} \in \mathcal{S}_{\mathrm{m}}}\left(c\left(\pi_{i}\right)\right) .
$$

Then the majority consistency measure is defined as

$$
\mathrm{Co}_{\mathrm{m}}\left(\pi_{i}\right)= \begin{cases}c_{\mathrm{m}}^{\min }-c\left(\pi_{i}\right), & c\left(\pi_{i}\right)<c_{\mathrm{m}}^{\min } \\ c\left(\pi_{i}\right)-c_{\mathrm{m}}^{\max }, & c\left(\pi_{i}\right)>c_{\mathrm{m}}^{\max } \\ 1, & \text { otherwise. }\end{cases}
$$

If any of the observations overlap, no majority observation is determined $\left(\left|\mathcal{S}_{\mathrm{m}}\right|=1\right)$. In this case an average consistency measure is determined, which utilises the weighted arithmetic mean. 
Definition 11. Weighted arithmetic mean: let $\boldsymbol{a}=\left(a_{i}\right)$ with $a_{i} \in \mathbb{R}$ and $i \in \mathbb{N}_{n}$ be a vector of input values, and $\boldsymbol{q}=\left(q_{i}\right)$ with $q_{i} \in \mathbb{R}$ a vector of corresponding weights. Then the weighted arithmetic mean is determined by

$$
\lambda_{\mathrm{WAM}}(\boldsymbol{q}, \boldsymbol{a})=\frac{\sum_{i=1}^{n} q_{i} \cdot a_{i}}{\sum_{i=1}^{n} q_{i}} .
$$

Then the average consistency measure is defined as

Definition 12. Average consistency measure (Glock et al., 2011): let $\pi_{i}$ be the observation of sensor $S_{i}$. For the remaining sensors $S_{j}$ with $j \neq i$, the vector $\pi^{*}=\left(\pi_{j} \mid j \neq\right.$ $i$ ) contains the respective observations, $\boldsymbol{r}^{*}=\left(r_{j} \mid j \neq i\right)$ contains the respective reliability measures after Eq. (5), and $\boldsymbol{v}^{*}=\left(v_{i, j} \mid j \neq i\right)$ contains the vicinity measures of observation $\pi_{i}$ to $\pi_{j}$, which is defined as

$$
v_{i, j}=1-\left|c\left(\pi_{i}\right)-c\left(\pi_{j}\right)\right|
$$

Then the average consistency measure is determined by

$$
\begin{aligned}
& \mathrm{Co}_{\mathrm{a}}\left(\pi_{i}\right)= \\
& \max \left(1-\max _{j=1 \mid j \neq i}^{n}\left(r_{j}\right), \lambda \operatorname{WAM}\left(\boldsymbol{r}^{*}, \boldsymbol{v}^{*}\right)\right),
\end{aligned}
$$

if and only if no majority observation is determined; hence $\left|\mathcal{S}_{\mathrm{m}}\right|=1$.

This measure determines the average of the vicinities between $\pi_{i}$ and $\pi_{j}$, weighed by the respective reliabilities $r_{j}$ in the case of high reliabilities $\left(r_{j} \rightarrow 1\right.$ for all $j$ ). If the other sensors are unreliable $\left(r_{j} \rightarrow 0\right.$ for all $\left.j\right)$, the observation of sensor $S_{i}$ is considered consistent to the truth such that $\mathrm{Co}_{\mathrm{a}}\left(\pi_{i}\right) \rightarrow 1$.

To summarise, the consistency measure for arbitrary observations is defined by

Definition 13. Consistency measure (Glock et al., 2011): let $\mathcal{S}_{\mathrm{m}}$ denote the set of sensors, which form the majority observation after Definition 9. Then the consistency measure is determined by

$$
\operatorname{Co}\left(\pi_{i}\right)= \begin{cases}\operatorname{Co}_{\mathrm{a}}\left(\pi_{i}\right), & \left|\mathcal{S}_{\mathrm{m}}\right|=1, \\ \mathrm{Co}_{\mathrm{m}}\left(\pi_{i}\right), & \text { otherwise }\end{cases}
$$

After introducing the concepts of majority observation and consistency, the dynamic sensor reliability is defined as

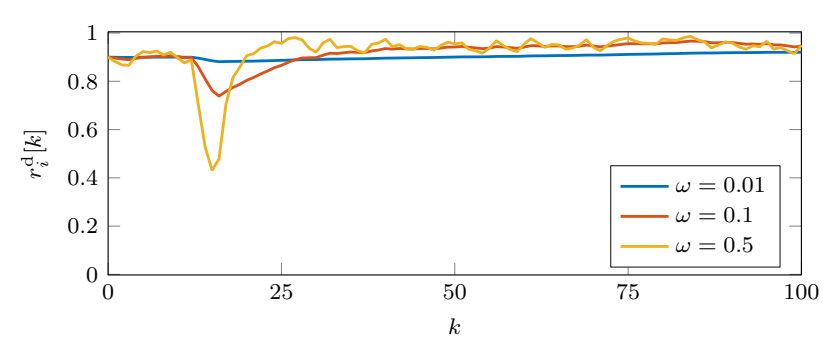

Figure 4. Development of the dynamic reliability $r_{i}^{\mathrm{d}}[k]$ of an exemplary sensor $S_{i}$ with $\omega=\{0.01,0.1,0.5\}$. At the beginning of the pictured period the measured value of the sensor drastically changes and is in conflict with other sensors.

Definition 14. Dynamic sensor reliability (Glock et al., 2011): let $\mathrm{Co}\left(\pi_{i}\right)$ be the consistency measure of the observation of sensor $S_{i} \in \mathcal{S}$. Then the dynamic sensor reliability at discrete time instance $k \in \mathbb{N}$ is determined as

$$
\begin{aligned}
& r_{i}^{\mathrm{d}}[k]=\omega \cdot \operatorname{Co}\left(\pi_{i}\right)+(1-\omega) \cdot r_{i}^{\mathrm{d}}[k-1], \\
& \text { with } r_{i}^{\mathrm{d}}[k]=1 \text { for all } k<0, \text { and } \omega \in[0,1] .
\end{aligned}
$$

Glock et al. (2011) defined the dynamic reliability measure in the form of an exponential moving averaging infinite impulse response filter (Meyer-Baese, 2007) to account for noise in the sensor observations and include information about the inertia of the monitored application by the smoothing factor $\omega$ : in order to react fast to changes in application with high inertia, the smoothing factor is set to $\omega \rightarrow 1$. In low-inertia applications, signal changes occur faster and thus demand $\omega \rightarrow 0$ in order to mitigate the influence of possible outliers in the adjustment of the sensor's reliability. An overview of the influence of $\omega$ on an exemplary dynamic sensor reliability is shown in Fig. 4. The smoothing effect of small values for $\omega$ is clearly visible.

\subsection{Sensor defect detection}

Sensor defects lead to sensor outputs, which do not represent the ground truth of the monitored system. In consequence, signals acquired by defective sensors result in information, which is in conflict with the information from intact sensors. These deliver signals, which represent the ground truth. Although the effects of conflicts in the input information is reduced by the $\mu_{\text {BalTLCS }}$ fusion algorithm applied in MACRO, additional detection of sensor defects can be utilised to identify and replace defective sensors. Then, conflicts between the acquired information vanish, which consequently leads to increased importance of the previously affected attributes.

In order to facilitate sensor defect detection, the approach of Glock et al. (2011) for monitoring sensor reliabilities is utilised (cf. Sect. 2.2). The reliability of sensor $S_{i}$ is determined on the basis of a static part $r_{i}^{\mathrm{s}}$ and a dynamic, hence 
time-dependent, part $r_{i}^{\mathrm{d}}[k]$. It is assumed that the sensor is reliable at the beginning of the monitoring and hence set to $r_{i}^{\mathrm{s}}=1$. If additional information is available regarding static reliability, this value may be adjusted. Since the dynamic part of the sensor reliability is time dependent, the whole measure is time dependent. Based on this, a sensor defect is detected, when its reliability measure $r_{i}[k]$ falls below a certain threshold:

Definition 15. Sensor defect decision rule: let $r_{i}[k]$ be the reliability of sensor $S_{i} \in \mathcal{S}$ at discrete time instance $k$. The average reliability of all sensors is computed as

$\bar{r}[k]=\frac{1}{n} \sum_{i=1}^{n} r_{i}[k]$.

Then a sensor defect is determined by evaluating the sensor defect decision rule:

$r_{i}[k]<\eta \cdot \bar{r}[k] \Rightarrow$ sensor $S_{i}$ is defective,

where $\eta \in[0,1]$ controls the decision threshold.

The decision threshold is designed variable with respect to $\bar{r}[k]$ to mitigate wrong decisions in real-world applications. If the monitored application changes its behaviour over time, this is not necessarily detected by all sensors at the same time. Hence, the observations of a subset of sensors become inconsistent and the respective reliabilities are decreased although no sensor defects occurred. After some time, all sensors that are contributing to one attribute, detect the change of the system leading to an equilibrated situation: sensor observations are consistent such that the previously decreased reliabilities increase again. If the decision threshold was constant in this case, a number of sensors would be declared as defective for some time and later as intact again.

Besides introducing a sensor defect decision rule, this contribution adapts the approach of Glock et al. (2011) to determine the individual reliabilities within groups of sensors.

Definition 16. Groupwise sensor reliability measure: the individual sensor reliability measure is determined on the basis of consistency evaluations among groups of sensors. These sensor groups are defined such that their sensors acquire signals influenced by the same property or constituent part of the monitored application. Each sensor group is a subset of all sensors denoted as $\mathcal{S}_{g} \subseteq \mathcal{S}$ with $g \in \mathbb{N}$, where an individual sensor $S_{i}$ is a member of one or more groups of sensors $\mathcal{S}_{g}$. Consequently, the groupwise sensor reliability measure is determined as

$$
r_{i}[k]=\frac{1}{G} \sum_{g=1}^{G} r_{i, g}[k],
$$

where $r_{i, g}$ is the sensor reliability measure of sensor $S_{i}$ in group $g$ determined after Eq. (5) with $\mathcal{S} \rightarrow \mathcal{S}_{g}$, and $G$ denotes the number of sensor groups $\mathcal{S}_{g}$, to which sensor $S_{i}$ is assigned.

This groupwise procedure is motivated due to several aspects:

- The sensors' signals inherit semantic or spatial proximities, as they are influenced by the same property (semantic proximity) or constituent part (spatial proximity). If the signals are influenced by a property, which is limited to one constituent part, semantic and spatial proximity occur at the same time.

- Due to said proximities, no coincidental correlations between independent signals occur. Causal relations between the signals inside one group are trustworthy.

- Applied within the context of MACRO, the required sensor groups are already defined as attributes. Hence, no further effort needs to be invested.

Although the application within the context of MACRO is beneficial, the approach is not restricted to it. It is applicable wherever grouping of sensors is possible. If no grouping is possible and all sensors need to be evaluated at once, the approach is also applicable: in this case only one group exists.

The approach of Glock et al. (2011) for monitoring sensor reliabilities is based on possibility distributions $\pi_{i}$, which model the sensor characteristics with respect to measurement uncertainties given output $x_{i}$. It is assumed to be available for each sensor in their approach. To the best knowledge of the authors, such information does not exist for any (non)commercially available sensor. Thus, it must be determined manually for each sensor in order to make the approach usable in real-world applications, for which the following practicable procedure is proposed:

Definition 17. Determination of sensor observation: the characteristics of sensor $S_{i}$ in terms of measurement uncertainty with respect to its current output $x_{i}$ is expressed by the probability density function (pdf) $p_{x_{i}}$.

If no other pdf is predetermined, it is assumed to be a uniform pdf on the interval $[a, b]$ :

$p_{x_{i}}(x)= \begin{cases}\frac{1}{b-a}, & a \leq x \leq b \\ 0, & \text { otherwise }\end{cases}$

The interval $[a, b]$ limits the maximum measurement error of sensor $S_{i}$ in case of $x_{i}$. It is either available from the sensor's data sheet, determined experimentally, or is approximated sensibly by an expert.

Then the statistical sensor characteristics function $p_{x_{i}}$ is transferred to the sensor observation $\pi_{i}$ by the truncated 
triangular probability-possibility transform (Lasserre et al., 2000; Mauris et al., 2000), which also allows for the transfer of Gaussian, triangular and Laplacian pdfs.

This procedure is carried out separately for each measurement $x_{i}$. The foundations of the truncated triangular probability-possibility transform are not included in this contribution since it is only applied and Lasserre et al. (2000) and Mauris et al. (2000) provided excellent introductions to it.

In order to determine the consistency measure for arbitrary sensors, their measurement scales are fuzzified before transforming $p_{x_{i}}$ to $\pi_{i}$.

Definition 18. Fuzzification of sensor measurement scales: with respect to MACRO, the fuzzification of the sensors' measurement scales is delivered through Modified-Fuzzy-Pattern-Classifier learning (cf. Definition 2) by ${ }^{\mathrm{N}} \mu_{i}: \mathbb{R} \rightarrow[0,1]$ for sensor $S_{i}$. Then the sensor characteristics function $p_{x_{i}}: \mathbb{R} \rightarrow[0,1]$ is transferred to ${ }^{\mathrm{N}} p_{x_{i}}:[0,1] \rightarrow[0,1]$ with

${ }^{\mathrm{N}} p_{x_{i}}\left({ }^{\mathrm{N}} \mu_{i}(x)\right)=p_{x_{i}}(x)$.

Consequently, the sensor observation $\pi_{i}: \mathbb{R} \rightarrow[0,1]$ is transferred to ${ }^{\mathrm{N}} \pi_{i}:[0,1] \rightarrow[0,1]$ with

$$
\mathrm{N}^{\mathrm{N}}{ }_{i}\left({ }^{\mathrm{N}} \mu_{i}(x)\right)=\pi_{i}(x) .
$$

The fuzzification through MFPC learning is already available as it is applied in MACRO for fusion on the attribute layer. Hence, the integration of arbitrary sensors is achieved in MACRO without any extra effort. In order to assist readability, ${ }^{\mathrm{N}} \pi_{i}={ }^{\mathrm{N}} \pi_{i}\left({ }^{\mathrm{N}} \mu_{i}(x)\right)$ is applied in the following. An exemplary sensor observation determined on fuzzified measurement scales is visualised in Fig. 5.

In addition, fuzzification has implications on the determination of the consistency index (cf. Eq. 6). Without fuzzification the whole range of real numbers is necessary to be evaluated $(x \in \mathbb{R})$, whereas due to fuzzification, the unit interval is evaluated $\left({ }^{\mathrm{N}} \mu_{i}(x) \in[0,1]\right)$.

From the following example, the necessity of an adaptation of the majority consistency measure as defined in Eq. (8) and Glock et al. (2011) is revealed.

Example 1. Properties of the majority consistency measure: regardless of the fuzzification of the measurement scale, let the centres of gravity of the following two observations be

$$
c\left(\pi_{1}\right)=c_{\mathrm{m}}^{\max }+\varepsilon, c\left(\pi_{2}\right)=c_{\mathrm{m}}^{\min }-\varepsilon,
$$

with $0<\varepsilon \ll 1$. Thus, both observations are close to the borders of the majority observation. Then the corresponding majority consistency measures are

$$
\operatorname{Co}_{\mathrm{m}}\left(\pi_{1}\right)=\varepsilon, \operatorname{Co}_{\mathrm{m}}\left(\pi_{2}\right)=\varepsilon .
$$

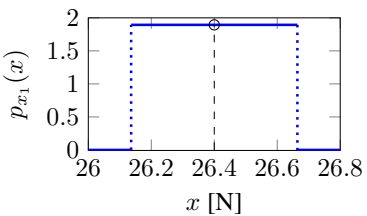

(a)

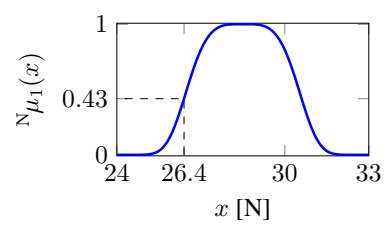

(c)

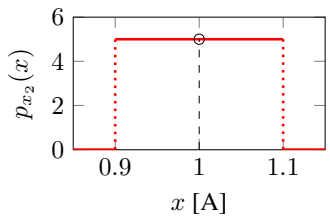

(b)

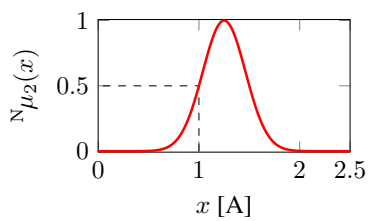

(d)

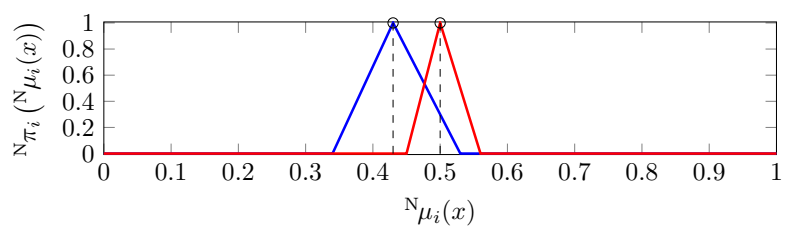

(e)

Figure 5. Exemplary determination of the sensor observations ${ }^{N} \pi_{1}$ and ${ }^{N} \pi_{2}$ on fuzzified measurement scales. The plots depict the respective functions of sensor $S_{1}$ measuring a force in $\mathrm{N}$ in blue, and those of sensor $S_{2}$ measuring an electric current in A in red. Note the incomparable measurement ranges and physical units, which are transformed step-by-step from (a) to (e) into a common space (a, b: sensor characteristics at measurement value $x_{i}$ (given by the dashed stem) represented by uniform probability density functions; $\mathbf{c}, \mathbf{d}$ : fuzzy membership functions ${ }^{\mathrm{N}} \mu_{i}$ modelling the normal condition as acquired by the respective sensor along with the fuzzified measurement value (given by the dashed line); e sensor observations as determined by the truncated triangular probability-possibility transform on the common fuzzified measurement scale along with the respective fuzzified measurement (given by the dashed stems)). The majority observation is visible in (e) in the overlapping region of the two functions.

Now let

$c\left(\pi_{1}^{\prime}\right)=c_{\mathrm{m}}^{\max }+\varepsilon^{\prime}, c\left(\pi_{2}^{\prime}\right)=c_{\mathrm{m}}^{\min }-\varepsilon^{\prime}$,

with $\varepsilon^{\prime}>\varepsilon$ being two observations further away from the majority observations' borders compared to $\pi_{1}$ and $\pi_{2}$. Then

$$
\mathrm{Co}_{\mathrm{m}}\left(\pi_{1}^{\prime}\right)=\varepsilon^{\prime}>\mathrm{Co}_{\mathrm{m}}\left(\pi_{1}\right), \mathrm{Co}_{\mathrm{m}}\left(\pi_{2}^{\prime}\right)=\varepsilon^{\prime}>\mathrm{Co}_{\mathrm{m}}\left(\pi_{2}\right) .
$$

The preceding example shows that the majority consistency measure increases with increasing distance of an observation to the majority observation. Contrarily, the majority consistency measure was defined in Glock et al. (2011) to be decreasing with increasing distance to the majority observation.

Glock et al. (2011) deduced $\mathrm{Co}_{\mathrm{m}}\left(\pi_{i}\right) \in[0,1)$ for all $i$. This is only fulfilled if the measurement scales of the sensors are fuzzified. Without fuzzification, observations with 
$c\left(\pi_{i}\right)=c_{\mathrm{m}}^{\max }+\Delta$, where $\Delta \in \mathbb{R}$, are valid and possible. This leads to $\mathrm{Co}_{\mathrm{m}}\left(\pi_{i}\right)>1$ for $\Delta>1$.

Therefore, the majority consistency measure is proposed to be adapted:

Definition 19. Adapted majority consistency measure: let $\mathrm{N}^{\mathrm{N}} \pi_{i}$ be the observation of sensor $S_{i} \in \mathcal{S}$ on a fuzzified scale according to Eq. (16). Then the adapted majority consistency measure is defined as

$$
\begin{aligned}
& \mathrm{Co}_{\mathrm{m}}\left({ }^{\mathrm{N}} \pi_{i}\right)= \\
& \begin{cases}1-\left(c_{\mathrm{m}}^{\mathrm{min}}-c\left({ }^{\mathrm{N}} \pi_{i}\right)\right), & c\left({ }^{\mathrm{N}} \pi_{i}\right)<c_{\mathrm{m}}^{\min }, \\
1-\left(c\left(^{\mathrm{N}} \pi_{i}\right)-c_{\mathrm{m}}^{\max }\right), & c\left({ }^{\mathrm{N}} \pi_{i}\right)>c_{\mathrm{m}}^{\max }, \\
1, & \text { otherwise, }\end{cases}
\end{aligned}
$$

with $\mathrm{Co}_{\mathrm{m}}\left({ }^{\mathrm{N}} \pi_{i}\right) \in[0,1]$ for all $i$. It is a measure, which decreases with increasing distance of an observation to the majority observation.

All necessary parts for sensor defect detection are now available. To summarise, the sensor defect detection approach proposed in this section

- is based on the sensor reliability monitoring approach presented in Glock et al. (2011);

- demands fuzzification of the measurement scales in all cases, which is delivered at no additional cost in the context of MACRO;

- determines observation consistency within groups of sensors, which are delivered at no additional cost in the context of MACRO;

- adapts the majority consistency measure defined in Glock et al. (2011).

\section{Experiments and results}

In this section, the defect detection results under normal operating conditions are presented first. Afterwards it is shown that the defect detection also works when the monitored system is not in a normal operating state. The capabilities of the sensor fault detection approach for different fault types are finally shown. The real-world use case is a hazardous material store from the research project itsowl-IGel (itsowl-IGel, 2015). Different types of sensors are applied, which are listed in Table 1.

For the detection of leakages, electro-optical switches are used; they are activated when the measuring tip is surrounded by liquid and are placed at the bottom of the hazardous material store. The sensors are combined into 17 attributes and are positioned on the inside, the air ducts (ventilation), and the outside of the hazardous material store. A schematic view of the store and the used sensors is given in Fig. 6.

The attributes are listed in Table A1. Some attributes are defined manually based on their location (e.g. ventilation in),
Table 1. List of all sensors applied to the hazardous material store.

\begin{tabular}{lrl}
\hline Sensor type & Quantity & Output \\
\hline Temperature & 14 & analog \\
Smoke detector & 4 & digital \\
Differential pressure switch & 1 & digital \\
Gas sensor & 1 & analog \\
Leakage detector & 2 & digital \\
\hline Overall & 22 & \\
\hline
\end{tabular}

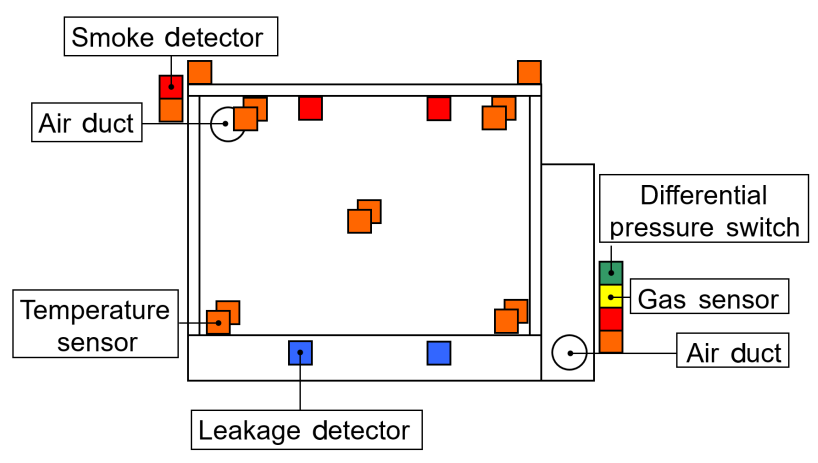

Figure 6. A schematic view of the hazardous material store with the sensors included. The different sensors are shown in different colours (temperature sensor: orange; smoke detector: red; leakage detector: blue; gas sensor: yellow; differential power switch: green). Additionally the air ducts are marked.

with some attributes also being defined based on their semantics (e.g. leakage). Others are a combination of the two aforementioned approaches (e.g. fire inside).

For the experiments, $\omega$ is set to 0.01 to avoid sudden changes in the computation of dynamic reliabilities $r_{i}^{\mathrm{d}}$ due to outliers (cf. Fig. 4 for $\omega=0.5$ ). The data and detailed configuration used for these experiments are available via Ehlenbröker et al. (2016a).

\subsection{Defect detection under normal operating conditions}

Sensor data were gathered in a hazardous material store demonstrator, which has been built for the itsowl-IGel project, under the following conditions: the hazardous material store was in a normal operating state with temperatures around $25^{\circ} \mathrm{C}$, whereas one temperature sensor (Temp_Inside_8) was delivering incorrect values (140 to $\left.150^{\circ} \mathrm{C}\right)$.

As can be seen in Fig. 7, the sensor defect is clearly detectable, as the sensor's reliability falls below the defect decision threshold.

The reliability value increases temporarily between 12:00 and 20:00 min because the temperature sensor randomly jumps back to values that are consistent with the other sensors in the hazardous material store. At the end of the ex- 


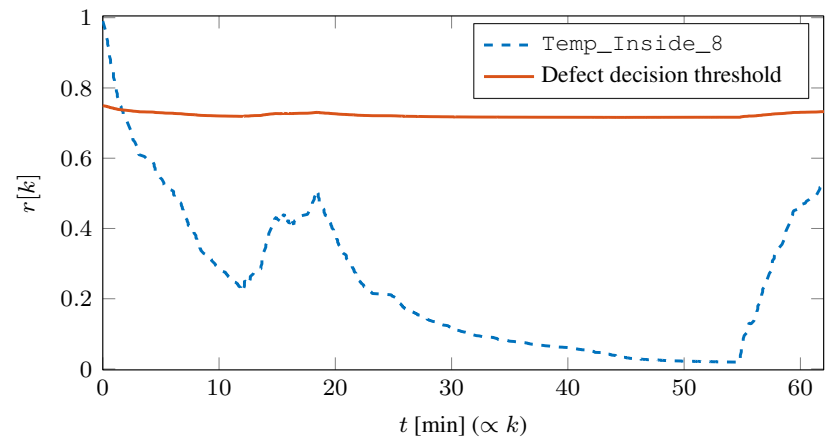

Figure 7. The defect decision threshold $(\eta=0.75)$ and the reliability $r[k]$ of the defective sensor Temp_Inside_8 with a random error $(\omega=0.01)$.

periment, the measurements of Temp_Inside_8 are again more consistent with the values of the other sensors and consequently the reliability increases.

\subsection{Defect detection during non-normal operating conditions}

To demonstrate that the detection of a sensor defect is also possible, if the hazardous material store is in a critical state, a smoke detector is falsely activated during an actual leakage in the store. Leakages occur, e.g., after a container with chemicals was damaged during the handling of the containers. As the stored chemicals are often toxic or flammable, it is important to reliably detect leakages, in spite of possible sensor defects.

The leakage in this scenario is an ongoing process: a damaged container releases its fluid content, which begins to gather itself at the bottom of the hazardous material store. At the same time parts of the leaking fluid evaporate and are detected by the gas sensor. The detection of the leaked fluids at the bottom of the store takes a longer time, as the leakage detectors only detect larger quantities of fluid.

The defective smoke detector was falsely activated after 3:00 min. This results in a steady decrease of this sensor's reliability, as displayed in Fig. 8. The defect of this sensor was detected at around 5:30 min, when the sensor's reliability crossed the defect decision threshold $\eta$.

Although Smoke_Inside_0 delivered incorrect values, the critical system state caused by the leakage is reliably represented in the decreasing system health ${ }^{\mathrm{N}} h[k]$ (cf. Eq. 4), as can be seen in Fig. 9.

The system health develops stepwise, with the first drop at about 3:00 min caused by the defect of the smoke detector. The following two drops are caused by the gas detector, which detects a gas leakage (at about 8:00 min), and the two leakage detectors activating at around 13:00 min. The visible jitter of the system health is caused by fluctuating measurements of the gas sensor.

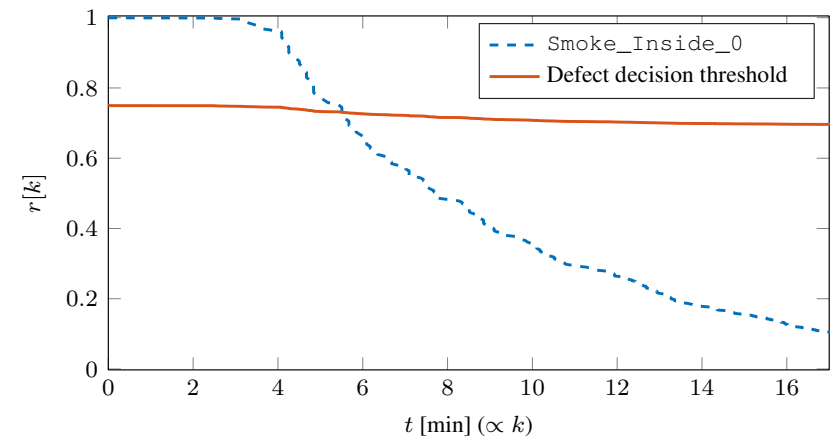

Figure 8. The defect decision threshold $(\eta=0.75)$ and the reliability $r[k]$ of the defective sensor Smoke_Inside_o $(\omega=0.01)$.

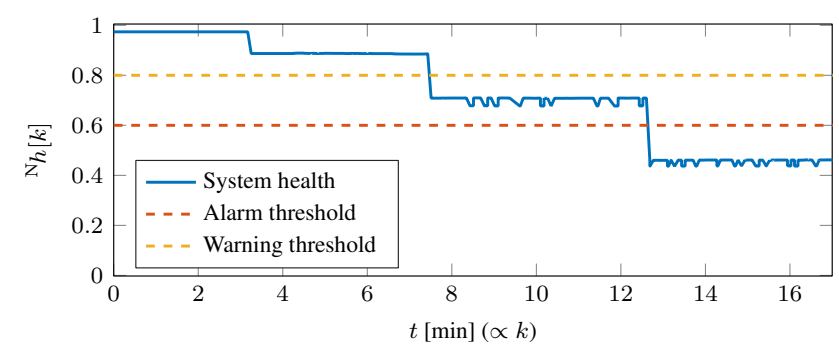

Figure 9. The system health ${ }^{\mathrm{N}} h[k]$ of the monitored system during a leakage over time. It is the aggregated result of the fusion process on system level (cf. Eq. 4) and represents the state of the store. The depicted warning and alarm thresholds were manually set. They are used for the generation of alarms for operators.

\subsection{Defect detection of different fault types}

In the following an overview about the detection of four sensor fault types with different strengths is given. For this evaluation, a multi-hour data recording of sensor Temp_Inside_2 from the hazardous material store in normal operation has been superposed with the following typical defects (cf. Helwig et al., 2015):

Peaks: Sensor reading outliers are simulated by adding $100^{\circ} \mathrm{C}$ at random time instances with rates between 1 and 10 peaks per minute.

Offset: Sensor values are evaluated at constant offsets between 1 and $5^{\circ} \mathrm{C}$.

Drift: Drifting sensor data are evaluated for constant rates between 1 and $5 \% \circ \mathrm{h}^{-1}$.

Noise: Additive zero-mean Gaussian noise is evaluated for signal-to-noise ratios (SNR) of 10 and $0 \mathrm{~dB}$.

Defect decision threshold $\eta$ and $\omega$ are set identical to the previously used values $(\eta=0.75, \omega=0.01)$. The data and detailed configuration used for these experiments is available via Ehlenbröker et al. (2016b). 


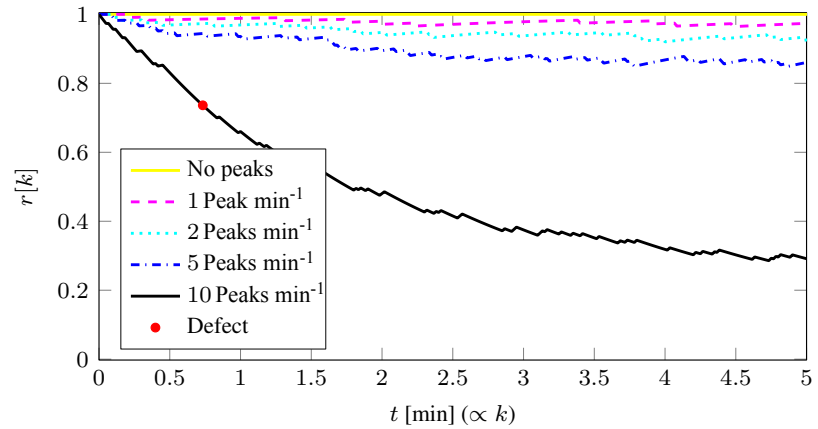

Figure 10. Reliability and detected defects for peak sensor faults. Peaks are randomly inserted to the measurement with frequencies from 1 to 10 peaks per minute by adding $100^{\circ} \mathrm{C}$ to the original sensor readings. The detected defect is marked by a red dot.

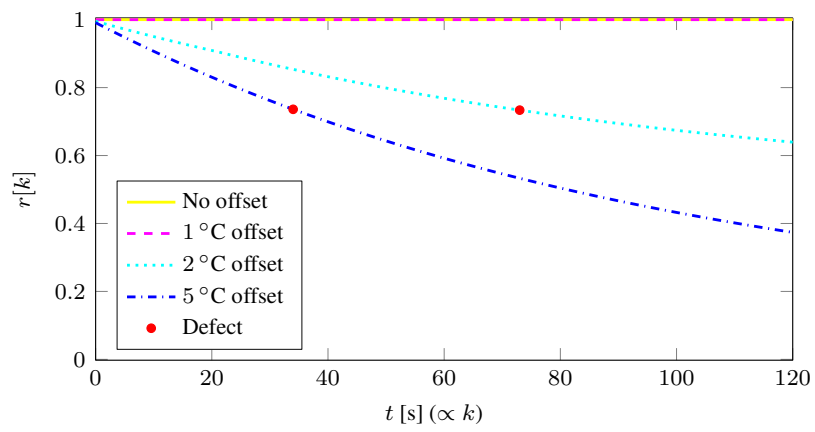

Figure 11. Reliability and detected defects for offset sensor faults. Results are shown for constant offsets between 1 and $5^{\circ} \mathrm{C}$. Detected defects are marked by a red dot.

The evaluation results in terms of sensor reliabilities are depicted in Figs. 10-13. For reference, the sensor reliability without a defect is included in each figure. It is visible that these fault-free cases are correctly detected to include no defect.

Figure 10 shows the performance of the proposed algorithm for peak errors. No defects are detected up to a peak frequency of 5 peaks per minute, whereas a defect is detected at 1 min running time at a level of 10 peaks per minute.

Results on offset faults are shown in Fig. 11. An offset of $1{ }^{\circ} \mathrm{C}$ remains undetected, whereas it takes around 75 and $35 \mathrm{~s}$ to detect 2 and $5^{\circ} \mathrm{C}$ offsets, respectively.

The defect detection behaviour for peaks and offset is due to the exponential averaging of the sensor reliability. It allows sensor behaviour deviations to a certain extent, which does not imply an actual defect. Its sensitivity is adjusted by $\omega$.

For drift errors, the detection is dependent on the length of the observed period: a drift fault of $5 \% \mathrm{~h}^{-1}$, as depicted in Fig. 12, is detected after $10 \mathrm{~h}$. The detection of a drift error of $2 \% o \mathrm{~h}^{-1}$ takes $16 \mathrm{~h}$. The drift fault of $1 \% \mathrm{oh}-1$ is not detected during the monitored time period.

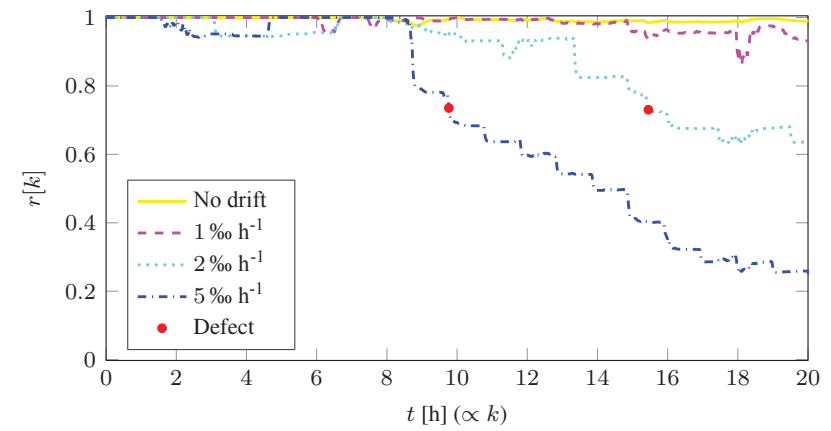

Figure 12. Reliability and detected defects for drift sensor faults. Results are shown for drift rates between 1 and $5 \% \circ \mathrm{h}^{-1}$. Detected defects are marked by a red dot.

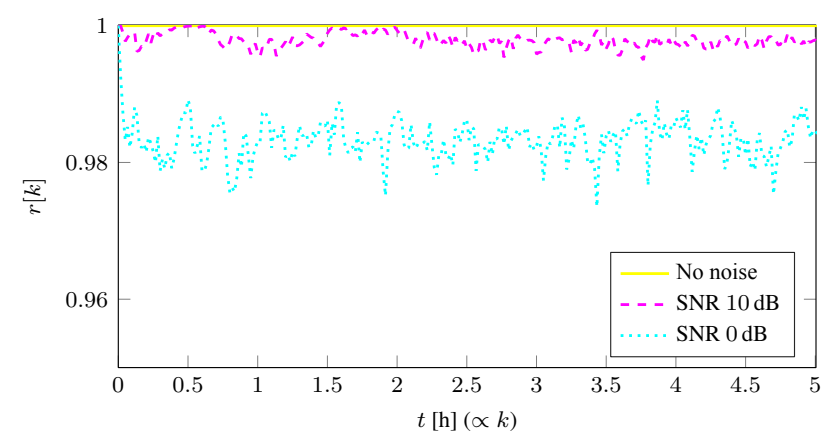

Figure 13. Reliability and detected defects for noise sensor faults. The evaluated signals contain additive zero-mean Gaussian noise with signal-to-noise ratios (SNR) of 10 and $0 \mathrm{~dB}$. No defect is detected.

Figure 13 depicts the results for noise faults. As is seen in the figure, no noise faults are detected. Compared to other sensor faults, the reliability decreases to $r=0.985$ on average for the signal containing maximal noise (SNR $0 \mathrm{~dB}$ ).

The detection behaviour for the latter two defect types attributed to the fact that the MACRO information fusion approach, on which this sensor defect approach is based. It is geared to be tolerant towards signal variations and noise in order to show stable behaviour to real-world system variations and prevent wring decisions. However, its sensitivity is adjusted by manual adjustments of the membership functions' widths $C_{i}$.

Compared to the results from Helwig et al. (2015), the proposed sensor defect detection is less sensitive towards the evaluated defect types. As mentioned before, the sensitivity is adjustable. However, the required sensitivity is dependent on the respective application. It has nevertheless been shown that defect detection is possible in general. 


\section{Conclusion and outlook}

This paper presents a method to generate a consistency-based reliability assessment for sensors, which is utilised to detect sensor defects. The approach is embedded into the information fusion system MACRO, which is well-suited for the implementation of the proposed sensor defect detection approach. As MACRO combines sensors in attributes, sensor groups with semantic and/or spatial proximity, which are primed to be used in the proposed consistency-based defect detection approach, are already employed. However, the approach is applicable also in the context of other information fusion applications. It was shown that the approach is capable of detecting sensor defects. The tests were carried out in the context of two real-world examples, in which a hazardous material store was monitored during normal operation and during a leakage. It was possible to detect the sensor defects in both scenarios. Additional tests for typical sensor defect types evaluate the defect detection approach with respect to peak, offset, drift, and noise. It is shown that defect detection is in general possible.

Sensor defects directly influence the conflict between sensor inputs. Hence, the conflict (which is the attribute's negated importance in the context of MACRO, delivered at no additional cost) seems to be an appropriate indicator for a possible sensor defect. It is to be investigated, whether this is exploitable to determine and assess sensor reliabilities only in cases, where the conflict exceeds a certain level. The presumably saved computational resources and effects on the defect detection's accuracy are to be evaluated. Further, a detailed look into more complex scenarios is of interest: defects of multiple sensors have not been considered, yet. If necessary, the proposed defect decision rule and the groupwise sensor reliability measure will be adapted. An investigation of false-positive and false-negative defect detection rates and the identification of optimisation possibilities of said rates needs also to be carried out. As the presented approach is insensitive to sensor faults of small values, an investigation of changes to detect additional sensor faults is also of importance.

\section{Data availability}

The data applied in this article are published on Zenodo as the two data sets "Sensor Defect Detection Datasets with Configuration" and "Typical Sensor Defects Dataset" under Creative Commons Attribution License and are free for everyone to download (Ehlenbröker et al., 2016a, b). 


\section{Appendix A: Attributes}

The list of all attributes and their associated sensors is presented in Table A1.

Table A1. List of all attributes and their associated sensors. Attributes are named according to their position (e.g. outside, inside up, ventilation in). Additionally, most attributes also include a monitored physical property (e.g. temperature) or semantics (e.g. fire).

\begin{tabular}{|c|c|}
\hline Attribute & Sensors \\
\hline Temperature outside & Temp_Outside_0,Temp_Outside_1, Temp_Ventilation_In \\
\hline Temperature inside up & Temp_Inside_0,Temp_Inside_1, Temp_Inside_2, Temp_Inside_3 \\
\hline Temperature inside down & Temp_Inside_6, Temp_Inside_7, Temp_Inside_8, Temp_Inside_9 \\
\hline Temperature inside & $\begin{array}{l}\text { Temp_Inside_0, Temp_Inside_1, Temp_Inside_2, Temp_Inside_3, } \\
\text { Temp_Inside_4, Temp_Inside_5, Temp_Inside_6, Temp_Inside_7, } \\
\text { Temp_Inside_8, Temp_Inside_9, Temp_Ventilation_Out }\end{array}$ \\
\hline Fire outside & $\begin{array}{l}\text { Smoke_Ventilation_In, Temp_Outside_0, Temp_Outside_1, } \\
\text { Temp_Ventilation_In }\end{array}$ \\
\hline Fire inside & $\begin{array}{l}\text { Temp_Inside_0, Temp_Inside_1, Temp_Inside_2, Temp_Inside_3, } \\
\text { Temp_Inside_4, Temp_Inside_5, Temp_Inside_6, Temp_Inside_7, } \\
\text { Temp_Inside_8, Temp_Inside_9, Temp_Ventilation_Out, Smoke_Inside_0, } \\
\text { Smoke_Inside_1, Smoke_Ventilation_Out }\end{array}$ \\
\hline Smoke emission inside & Smoke_Inside_0,Smoke_Inside_1, Smoke_Ventilation_Out \\
\hline Gas leak inside & Gas_Ventilation_Out \\
\hline Leakage inside & Gas_Ventilation_Out, Leakage_0, Leakage_1 \\
\hline Ventilation in & Smoke_Ventilation_In, Temp_Ventilation_In \\
\hline Ventilation out & $\begin{array}{l}\text { Gas_Ventilation_Out, } \\
\text { Diff_Pressure_Switch_Pressure_Ventilation_Out, } \\
\text { Smoke_Ventilation_Out, Temp_Ventilation_Out }\end{array}$ \\
\hline Air exchange & Diff_Pressure_Switch_Ventilation_Out \\
\hline Fire container up & $\begin{array}{l}\text { Temp_Inside_0, Temp_Inside_1, Temp_Inside_2, Temp_Inside_3, } \\
\text { Smoke_Inside_0, Smoke_Inside_1 }\end{array}$ \\
\hline Fire inside left & $\begin{array}{l}\text { Temp_Inside_1, Temp_Inside_3, Temp_Inside_6, Temp_Inside_8, } \\
\text { Smoke_Inside_0 }\end{array}$ \\
\hline Fire inside right & $\begin{array}{l}\text { Temp_Inside_0, Temp_Inside_2, Temp_Inside_7, Temp_Inside_9, } \\
\text { Smoke_Inside_1 }\end{array}$ \\
\hline Temperature inside down left & Temp_Inside_6, Temp_Inside_8 \\
\hline Temperature inside down right & Temp_Inside_7, Temp_Inside_9 \\
\hline
\end{tabular}


Acknowledgements. This article is based on the SENSOR 2015 conference contribution by the same authors (Ehlenbröker et al., 2015). The authors would like to thank Denis Petker for the helpful remarks and valuable help during the preparation of this contribution. Additionally, we would like to thank Udo Roth and the Denios AG for the development and provisioning of the hazardous material store demonstrator, and their support during the real-world tests. This work was partly funded by the German Federal Ministry of Education and Research (BMBF) under grant agreement no. 02PQ2112, within the Leading-Edge Cluster "Intelligent Technical Systems OstWestfalenLippe" (it's OWL).

Edited by: K.-D. Sommer

Reviewed by: three anonymous referees

\section{References}

Alpaydın, E.: Introduction to Machine Learning, Adaptive computation and machine learning, MIT Press, Cambridge, MA, 2nd Edn., 2010.

Bay, S. D. and Schwabacher, M.: Mining distance-based outliers in near linear time with randomization and a simple pruning rule, in: The Ninth ACM SIGKDD International Conference, edited by: Senator, T., Domingos, P., Faloutsos, C., and Getoor, L., p. 29, doi:10.1145/956750.956758, 2003.

Bishop, C. M.: Pattern recognition and machine learning, Information science and statistics, Springer, New York, NY, 8th Edn., 2009.

Choi, S. W., Lee, C., Lee, J.-M., Park, J. H., and Lee, I.B.: Fault detection and identification of nonlinear processes based on kernel PCA, Chemometr. Intell. Lab., 75, 55-67, doi:10.1016/j.chemolab.2004.05.001, 2005.

Dempster, A. P.: Upper and lower probabilities induced by a multivalued mapping, Ann. Math. Stat., 38, 325-339, doi:10.1214/aoms/1177698950, 1967.

Dubois, D. and Prade, H.: Fuzzy sets and probability: misunderstandings, bridges and gaps, 2nd IEEE International Conference on Fuzzy Systems, 2, 1059-1068, doi:10.1109/FUZZY.1993.327367, 1993.

Duquet, S.: Smart Sensors: Enabling Detection and Ranging for the Internet of Things and Beyond, Elektronik Praxis, April 2015.

Ehlenbröker, J.-F., Mönks, U., Wesemann, D., and Lohweg, V.: Condition Monitoring for Hazardous Material Storage, 19th IEEE Int. Conf. on Emerging Technologies and Factory Automation (ETFA 2014), Barcelona, Spain, 16-19 September 2014, 14, doi:10.1109/ETFA.2014.7005264, 2014.

Ehlenbröker, J.-F., Mönks, U., and Lohweg, V.: Consistency Based Sensor Defect Detection, in: SENSOR 2015, AMA Service GmbH, Nürnberg, Germany, 19-21 May 2015, 878-883, doi:10.5162/sensor2015/P10.4, 2015.

Ehlenbröker, J.-F., Mönks, U., and Lohweg, V.: Sensor Defect Detection Datasets with Configuration, Zenodo, doi:10.5281/zenodo.48728, 2016a.

Ehlenbröker, J.-F., Mönks, U., and Lohweg, V.: Typical Sensor Defects Dataset, Zenodo, doi:10.5281/zenodo.56358, 2016 b.

Elouedi, Z., Mellouli, K., and Smets, P.: Assessing Sensor Reliability for Multisensor Data Fusion Within the Transferable Belief Model, IEEE T. Syst. Man. Cy. B, 34, 782-787, doi:10.1109/TSMCB.2003.817056, 2004.
Glock, S., Voth, K., Schaede, J., and Lohweg, V.: A Framework for Possibilistic Multi-source Data Fusion with Monitoring of Sensor Reliability, in: World Conference on Soft Computing, San Francisco, USA, 23-26 May, 2011.

Hall, D. L. and Llinas, J. (Eds.): Handbook of Multisensor Data Fusion - The electrical engineering and applied signal processing series, CRC Press, Boca Raton FL, http://site.ebrary.com/lib/ alltitles/docDetail.action?docID=10142823, 2001a.

Hall, D. L. and Llinas, J.: Multisensor Data Fusion, in: Handbook of Multisensor Data Fusion, edited by: Hall, D. L. and Llinas, J., pp. CRC Press, Boca Raton FL, 1-1-1-10, doi:10.1201/9781420038545.pt1, 2001b.

Helwig, N., Pignanelli, E., and Schütze, A.: Detecting and Compensating Sensor Faults in a Hydraulic Condition Monitoring System, in: SENSOR 2015, AMA Service GmbH, Nürnberg, Germany, 1-21 May 2015, 641-646, doi:10.5162/sensor2015/D8.1, 2015.

Hu, Y., Chen, H., Xie, J., Yang, X., and Zhou, C.: Chiller sensor fault detection using a self-Adaptive Principal Component Analysis method, Energ. Buildings, 54, 252-258, doi:10.1016/j.enbuild.2012.07.014, 2012.

IEEE Computer Society: IEEE Standard Computer Dictionary: A Compilation of IEEE Standard Computer Glossaries - IEEE Std 610-1990, IEEE, New York, NY, USA, doi:10.1109/IEEESTD.1991.106963, 1990.

itsowl-IGel: Intelligentes Frühwarnsystem für Gefahrstofflager (Intelligent early warning system for hazardous material storage areas): Research Project sponsored by the Federal Ministry of Education and Research, available at: http://www.its-owl.de/projekte/innovationsprojekte/details/ intelligentes-fruehwarnsystem-fuer-gefahrstofflager/, 2015.

Jaynes, E. T.: Probability Theory: The Logic of Science, Cambridge Univ. Press, Cambridge, 727 pp., 2003.

Kerschen, G., Boe, P. D., Golinval, J.-C., and Worden, K.: Sensor validation using principal component analysis, VTT Symp., 14, 36 pp., doi:10.1088/0964-1726/14/1/004, 2005.

Khaleghi, B., Khamis, A., Karray, F. O., and Razavi, S. N.: Multisensor data fusion: A review of the state-of-the-art, Inform. Fusion, 14, 28-44, doi:10.1016/j.inffus.2011.08.001, 2011.

Klir, G. J. and Yuan, B.: Fuzzy Sets and Fuzzy Logic: Theory and Applications, Pentice Hall, New Jersey, 1995.

Krüger, M.: Gradual vs. binary conflicts in Bayesian networks applied to sensor failure detection, 18th International Conference on Information Fusion, 2015, Washington, DC, 6-9 July 2015, 66-73, 2015.

Kusiak, A. and Song, Z.: Sensor Fault Detection in Power Plants, J. Energ. Eng.-ASCE, 135, 127-137, doi:10.1061/(ASCE)07339402(2009)135:4(127), 2009.

Larsen, H. L.: Importance weighted OWA aggregation of multicriteria queries, 18th International Conference of the North American Fuzzy Information Processing Society (NAFIPS 1999), New York, NY, 10-12 Jun 1999, 740-744, doi:10.1109/NAFIPS.1999.781792, 1999.

Larsen, H. L.: Efficient importance weighted aggregation between min and max, 9th International Conference on Information Processing and Management of Uncertainty in Knowledge-Based Systems (IPMU 2002), Annecy, France, 1-5 July 2002, 740-744, 2002. 
Lasserre, V., Mauris, G., and Foulloy, L.: A simple possibilistic modelisation of measurement uncertainty, in: Uncertainty in Intelligent and Information Systems, edited by: Bouchon-Meunier, B., Yager, R. R., and Zadeh, L. A., World Scientific, 58-69, doi:10.1142/9789812792563_0005, 2000.

Lohweg, V. and Mönks, U.: Sensor Fusion by Two-Layer Conflict Solving, 2nd International Workshop on Cognitive Information Processing (CIP 2010), Elba, Italy, 14-16 June 2010, 370-375, doi:10.1109/CIP.2010.5604094, 2010a.

Lohweg, V. and Mönks, U.: Fuzzy-Pattern-Classifier Based Sensor Fusion for Machine Conditioning, in: Sensor Fusion and its Applications, edited by: Thomas, C., InTech, 319-346, doi:10.5772/9969, 2010b.

Lohweg, V., Diederichs, C., and Müller, D.: Algorithms for Hardware-Based Pattern Recognition, EURASIP J. Adv. Sig. Pr., 2004, 1912-1920, doi:10.1155/S1110865704404247, 2004.

Luo, R. C. and Kay, M. G.: Multisensor integration and fusion in intelligent systems, IEEE Transactions on Systems, Man, and Cybernetics, 19, 901-931, doi:10.1109/21.44007, 1989.

Martin, A., Jousselme, A.-L., and Osswald, C.: Conflict measure for the discounting operation on belief functions, 11th International Conference on Information Fusion, 2008, Cologne, Germany, 30 June-3 July 2008, 1-8, 2008.

Mattern, D., Jaw, L., Guo, T.-H., Graham, R., and McCoy, W.: Using neural networks for sensor validation, in: 34th AIAA/ASME/SAE/ASEE Joint Propulsion Conference and Exhibit, Cleveland, OH, 13-15 July 1998, doi:10.2514/6.19983547, 1998.

Mauris, G., Lasserre, V., and Foulloy, L.: Fuzzy Modeling of Measurement Data Acquired from Physical Sensors, IEEE Transactions on Instrumentation and Measurement, 49, 1201-1205, doi:10.1109/19.893256, 2000.

Mehranbod, N., Soroush, M., Piovoso, M., and Ogunnaike, B. A.: Probabilistic model for sensor fault detection and identification, AIChE Journal, 49, 1787-1802, doi:10.1002/aic.690490716, 2003.

Mehranbod, N., Soroush, M., and Panjapornpon, C.: A method of sensor fault detection and identification, J. Process. Contr., 15, 321-339, doi:10.1016/j.jprocont.2004.06.009, 2005.

Meyer-Baese, U.: Digital signal processing with field programmable gate arrays, Signals and communication technology, Springer, Berlin, New York, 3rd Edn., 2007.

Mönks, U. and Lohweg, V.: Machine Conditioning by Importance Controlled Information Fusion, IEEE 18th Conference on Emerging Technologies \& Factory Automation (ETFA), Cagliari, Italy, 10-13 September 2013, 1-8, doi:10.1109/ETFA.2013.6647984, 2013.

Mönks, U. and Lohweg, V.: Fast Evidence-based Information Fusion, 4th International Workshop on Cognitive Information Processing (CIP), Copenhagen, Denmark, 26-28 May 2014, 1-6, doi:10.1109/CIP.2014.6844508, 2014.

Mönks, U., Petker, D., and Lohweg, V.: Fuzzy-Pattern-Classifier Training with Small Data Sets, in: Information Processing and Management of Uncertainty in Knowledge-Based Systems, 13th International Conference, IPMU 2010, Dortmund, Germany, 28 June-2 July 2010, 426-435, doi:10.1007/978-3-642-14055$6 \_44,2010$.
Mönks, U., Voth, K., and Lohweg, V.: An Extended Perspective on Evidential Aggregation Rules in Machine Condition Monitoring, 3rd International Workshop on Cognitive Information Processing (CIP), Baiona, Spain, 28-30 May 2012, 1-6, doi:10.1109/CIP.2012.6232905, 2012.

Mönks, U., Trsek, H., Dürkop, L., Geneiß, V., and Lohweg, V.: Towards distributed intelligent sensor and information fusion, Mechatronics, 34, 63-71, doi:10.1016/j.mechatronics.2015.05.005, 2015.

Niederhöfer, M. and Lohweg, V.: Application-based approach for automatic texture defect recognition on synthetic surfaces, IEEE International Conference on Emerging Technologies and Factory Automation, Hamburg, Germany, 15-18 September 2008, 229232, doi:10.1109/ETFA.2008.4638397, 2008.

Ricquebourg, V., Delahoche, L., Marhic, B., Delafosse, M., JollyDesodt, A. M., and Menga, D.: Anomalies recognition in a context aware architecture based on TBM approach, 11th International Conference on Information Fusion, Cologne, Germany, 30 June-3 July 2008, 1-8, 2008.

Ross, A. and Jain, A. K.: Multimodal Human Recognition Systems, in: Multi-Sensor Image Fusion and Its Applications, edited by: Liu, Z. and Blum, R., CRC Press, 289-301, doi:10.1201/9781420026986.ch9, 2005.

Shafer, G.: A Mathematical Theory of Evidence, Princeton University Press, Princeton, NJ, 1976.

Sharma, A. B., Golubchik, L., and Govindan, R.: Sensor Faults: Detection Methods and Prevalence in Real-World Datasets, ACM T Sensor Network, 6, 1-39, doi:10.1145/1754414.1754419, 2010.

Steinberg, A. N. and Bowman, C. L.: Revisions to the JDL Data Fusion Model, in: Handbook of Multisensor Data Fusion, edited by: Hall, D. L. and Llinas, J., CRC Press, Boca Raton FL, 2-12-19, doi:10.1201/9781420038545.ch2, 2001.

Xu, X., Hines, J. W., and Uhrig, R. E.: Sensor Validation and Fault Detection Using Neural Networks, Proc. Maintenance and Reliability Conference (MARCON 99), Gatlinburg, Tennessee, 10-12 May 1999, 10-12, 1999.

Yager, R. R.: On the dempster-shafer framework and new combination rules, Inform. Sciences, 41, 93-137, doi:10.1016/00200255(87)90007-7, 1987.

Yager, R. R.: On ordered weighted averaging aggregation operators in multicriteria decisionmaking, IEEE T. Syst. Man Cyb. 18, 183-190, doi:10.1016/B978-1-4832-1450-4.50011-0, 1988.

Zadeh, L. A.: Fuzzy Sets, Inform. Control, 8, 338-353, doi:10.1016/S0019-9958(65)90241-X, 1965.

Zadeh, L. A.: Fuzzy sets as a basis for a theory of possibility, Fuzzy Set. Sys., 1, 3-28, doi:10.1016/0165-0114(78)90029-5, 1978.

Zadeh, L. A.: A simple view of the Dempster-Shafer theory of evidence and its implication for the rule of combination, in: Fuzzy Sets, Fuzzy Logic, and Fuzzy Systems, 674-679, doi:10.1142/9789814261302_0033, 1986.

Zhu, Y., Jin, X., and Du, Z.: Fault diagnosis for sensors in air handling unit based on neural network preprocessed by wavelet and fractal, Energ. Buildings, 44, 7-16, doi:10.1016/j.enbuild.2011.09.043, 2012. 